\title{
Comparaison de la composition physicochimique du lait camelin et bovin du Sud tunisien; variation du pH et de l'acidité à différentes températures
}

Amel SBOUI ${ }^{1,3 *}$, Touhami KHORCHANI', Mongi DJEGHAM. ${ }^{2}$ et Omrane BELHADJ ${ }^{3}$

'Laboratoire d'Elevage et de la Faune Sauvage, Institut des Régions Arides, Médenine Tunisie, route elfgè 4119, Médenine, Tunisie.

2Laboratoire de Physiologie thérapeutique, Ecole Nationale de Médecine Vétérinaire

Sidi Thabet Tunisie

${ }^{3}$ Laboratoire de Biochimie et Techno Biologie, Faculté des Sciences de Tunis, Tunisie

*Correspondance,courriel : amelsb6@yahoo.fr

\section{Résumé}

La composition physicochimique du lait de chamelle est variable selon l'alimentation des animaux, les conditions environnementales ainsi que la période de lactation.

Dans le but de déterminer la composition du lait de chamelle du sud tunisien, le lait camelin de la région a été comparé à celui bovin sur le plan de l'acidité et du pH du lait des deux espèces en fonction de la température du milieu.

Le lait de chamelle est plus acide $\left(17,25 \pm 1,035^{\circ} \mathrm{D}\right)$ et moins dense $(d=1,027 \pm$ $0,003)$ que le lait de vache. Le lait de vache est plus riche en matière protéique $(34,15 \pm 3,11 \mathrm{~g} / \mathrm{L})$, matière sèche $(119,43 \pm 15,34 \mathrm{~g} / \mathrm{L})$ et azote non protéique NPN $(1,04 \pm 0,08 \mathrm{~g} / \mathrm{L})$.

Le suivi de la variation du $\mathrm{pH}$ et de l'acidité du lait camelin et bovin à trois températures différentes (température ambiante $\left(25^{\circ} \mathrm{C}\right)$, à $4^{\circ} \mathrm{C}$ et à $-4^{\circ} \mathrm{C}$ ) a révélé une acidification plus lente du lait de chamelle dans les trois cas avec une conservation durant 30 heures à température ambiante, 7 jours dans le réfrigérateur $\left(4^{\circ} \mathrm{C}\right)$ alors quelle est de 5 jours pour le lait de vache à la même température. La congélation du lait à $-4^{\circ} \mathrm{C}$ peut ainsi constituer une méthode simple pour la conservation du lait camelin qui n'est accessible que dans les régions de sa production.

Mots-clés : Lait, chamelle, vache, composition physicochimique, acidité, température 


\section{Abstract \\ Comparison of the gross composition of Tunisian camel and cow milk; Variations of $\mathrm{pH}$ and acidity at various temperatures}

The gross composition camel milk is variable according to the food of the animals, the environmental conditions as well as the period of lactation.

This study is aiming to determine the composition of camel milk from south of Tunisia in comparison with cow milk and to follow the variations of theirs $\mathrm{pH}$ and acidity at various temperature.

The physical analysis of milk showed that camel milk is more acid and less dense than the cow milk. Its viscosity $(3.21 \pm 0.41)$ is significantly higher than that of bovine milk (2.46 \pm 0.53$)$.

The chemical composition showed that bovine milk is richer in protein $(34.15 \pm 3.114$ $\mathrm{g} / \mathrm{L})$, dry matter $(119.43 \pm 15.34 \mathrm{~g} / \mathrm{L})$ and Non Protein N (NPN) $(1.04 \pm 0.08 \mathrm{~g} / \mathrm{L})$. The follow-up of $\mathrm{pH}$ and acidity variations of milk from the two at three different temperatures (ambient temperature $\left(25^{\circ} \mathrm{C},+4^{\circ} \mathrm{C}\right.$ and $-4^{\circ} \mathrm{C}$ ) revealed a slower acidification of camel milk ( 7 days at $\left(4^{\circ} \mathrm{C}\right)$ ).

On the other hand frozen camel or cow milk can be conserved several weeks and thus constitute a simple method for storing up milk especially camel milk which is accessible only in arid lands.

Keywords : milk, camel, cow, gross composition, acidity, temperature

\section{Introduction}

Le lait de chamelle, comme celui des autres mammifères, est un milieu de composition chimique et physique complexe qui permet au jeune chamelon de couvrir ses besoins énergétiques et nutritionnels pendant la première étape de son existence. Ce milieu est toutefois éminemment périssable par suite de sa forte teneur en eau, de son pH voisin de la neutralité et de sa richesse en lactose qui le rendent rapidement altérable par voie microbienne et par voie enzymatique. Par ailleurs, la fragilité de ses équilibres physico-chimiques (émulsion de matière grasse, suspension colloïdale de protéines) peut conduire facilement à une déstabilisation par voie physique, en particulier sous l'action de chocs mécaniques et thermiques.

Le lait camelin possédait un certain nombre de particularités de composition chimique et physique qui peuvent influencer son aptitude à la conservation. 
Dans ce cadre, nous avons procédé à un suivi de la composition physicochimique du lait de chamelle du sud tunisien ainsi que la variation de son $\mathrm{pH}$ et acidité à différentes températures, tout en le comparant avec le lait de vache élevée dans la même région.

\section{Matériel et méthodes}

\section{2-1. Origine du lait utilisé}

Le lait de chamelle (L.Ch) analysé est un mélange de lait de plusieurs femelles appartenant au troupeau de dromadaires élevés à l'Institut des Régions Arides de Médenine, Tunisie.

Les échantillons du lait de vache (L.V) sont collectés d'un troupeau de vaches élevées dans la même région du sud tunisien.

\section{2-2. Prélèvements}

L'analyse a porté sur 20 échantillons de lait de chaque espèce, prélevés des femelles en début de lactation ( $2^{\text {ème }}$ et $3^{\text {ème }}$ mois). La traite des animaux est effectuée le matin avant la sortie du troupeau au pâturage.

Toutes les analyses physicochimiques sont réalisées dans le Laboratoire d'Elevage et de Faune Sauvage de l'Institut des Régions Arides de Médenine.

\section{2-3. Répartition des échantillons}

Les échantillons du lait frais sont mis à température du laboratoire après détermination du pH et de l'acidité à l'état frais. Ces deux paramètres sont mesurés le matin et l'après midi jusqu'à acidification du lait. Les échantillons du lait sont conservés dans le réfrigérateur à $4^{\circ} \mathrm{C}$ pour suivre l'évolution du pH et de l'acidité d'une façon journalière. Le suivi est arrêté lorsqu'on remarque une modification de l'aspect du lait : coagulation et odeur désagréable.

Congelés à $-4^{\circ} \mathrm{C}$ pendant trois semaines à partir du jour de prélèvement, les mêmes paramètres physiques sont analysés pour étudier leur stabilité des échantillons à cette température de congélation. 


\section{2-4. Méthodes d'analyses}

Les paramètres chimiques déterminés sont la teneur en matière sèche ou extrait sec total, la teneur en cendres totales, la matière grasse, lactose, azote non protéique (NPN), protéines totales et en éléments minéraux, à savoir $\mathrm{Mg}, \mathrm{Na}, \mathrm{K}$, Ca, Cuivre (Cu) et $\operatorname{Zinc}(\operatorname{Zn})$.

Trois paramètres physiques sont de routine évalués. II s'agit du pH, de l'acidité et de la densité de chaque échantillon de lait.

\section{2-4-1. Mesure du pH et de l'acidité}

Ces deux paramètres sont mesurés dès la traite des animaux sur le mélange de lait de chaque espèce. Le pH est mesuré à $20^{\circ} \mathrm{C}$ à l'aide d'un $\mathrm{pH}$ mètre de type ThermoOrion et l'acidité est dosée par titration avec une solution de $\mathrm{NaOH}$ et exprimée en ${ }^{\circ}$ Dornic.

\section{2-4-2. Densité}

La densité est déterminée à l'aide d'un thermo lactodensimètre PAAR DMA 35. Elle est toujours mesurée à $20^{\circ} \mathrm{C}$.

\section{2-4-3. Matière sèche et cendres}

La matière sèche, exprimée en gramme par litre de lait, est calculée après pesée de l'échantillon à $100^{\circ} \mathrm{C}$ pendant 7 heures de son résidu sec. Les prises d'essai sont de $5 \mathrm{~g}$. La teneur en cendres, exprimée en $\mathrm{g} / \mathrm{L}$ de lait, est déterminée après dessiccation à $505^{\circ} \mathrm{C}$.

\section{2-4-4. Matière grasse}

La méthode suivie est une méthode acido-butyrométrique en utilisant II neusol solution II citée par Farah [1]. Cette méthode repose sur la lecture directe sur un butyromètre de la quantité de matière grasse contenue dans $12 \mathrm{~mL}$ de l'échantillon après une centrifugation en présence de l'alcool amylique. La lecture directe des graduations détermine la quantité de matière grasse en $\mathrm{g} / \mathrm{L}$.

\section{2-4-5. Dosage du lactose}

Le lactose est exprimé en g/L et déterminé par la méthode de Bertrand [2]. 


\section{2-4-6. Matière azotée totale}

L'azote total (NT) et l'azote non protéique (NNP) sont dosés par la méthode de Kjeldahl $(N \times 6,38)[2]$ après minéralisation sur unité Buchi 425 , distillation sur une unité Buchi 320 (Buchi Laboratoriums-Tchnik, Flawil, Switzerland) et titration avec l'acide chlorhydrique $0,1 \mathrm{~N}$.

\section{2-4-7. Éléments minéraux}

Le dosage des élément minéraux est réalisé à l'aide d'un spectrophotomètre d'absorption atomique de type Hitachi: Shimadzu AA- 6800, en présence du chlorure de lanthane pour le dosage du Calcium (Ca) et Magnésium (Mg) (Sigma Chemical Co ; St Louis USA) et du chlorure de césium (Merck) pour le Sodium (Na) et Potassium (K).

\section{2-5. Analyse statistique}

Pour comparer l'effet des différents facteurs étudiés sur les échantillons de laits utilisés on a eu recours au test T de Student à $5 \%$ sur Exel $2003(p=0,05)$.

Toutes les analyses sont faites en double pour chaque échantillon et les valeurs représentées dans les tableaux et les courbes sont les moyennes des résultats obtenus pour les 8 échantillons déjà décrits.

\section{Résultats et discussion}

\section{3-1. Comparaison entre le lait de vache et le lait de chamelle}

\section{3-1-1. Caractéristiques organoleptiques}

Le lait de chamelle est de couleur blanche mate, goût un peu salé et d'un aspect plus visqueux que le lait de vache, qui est de couleur jaunâtre. Ces caractéristiques et surtout le goût du lait de chamelle diffère selon l'alimentation des animaux et la disponibilité en eau [3]. L'ingestion de fourrages comme la luzerne, donne un goût sucré, certaines plantes halophytes le rendent salé $[4,5]$. Dans notre cas le pâturage est riche en plantes halophiles d'où le goût salé du lait. 


\section{3-1-2. paramètres physiques}

Les résultats de l'analyse physique sont illustrés dans le Tableav I l'analyse statistique ne montre aucune différence significative entre les laits des espèces.

Tableau 1 : Comparaison des paramètres physiques du lait de vache et de chamelle

\begin{tabular}{|l|c|c|c|c|c|}
\hline \multirow{2}{*}{ Paramètres } & \multicolumn{2}{|c|}{ Lait de chamelle (L.Ch) } & \multicolumn{2}{c|}{ Lait de vache (L.V) } & Test de T : \\
\cline { 2 - 5 } & Moyenne & Ecart-type & Moyenne & Ecart-type & valeur de p \\
\hline pH & 6,41 & 0,18 & 6,56 & 0,24 & 0,13 \\
\hline Acidité( $\left.{ }^{\circ} \mathrm{D}\right)$ & 17,2 & 1,03 & 17,12 & 0,64 & 0,81 \\
\hline Densité & 1,02 & 0,0032 & 1,028 & 0,0006 & 0,62 \\
\hline
\end{tabular}

$p \leq 0,05$ : La différence est significative entre les deux paramètres comparés $p>0,05$ : La différence est non significative

\section{3-1-3. Composantes chimiques}

La composition chimique du lait de chamelle parait plus riche que celle du lait de vache ce qui n'est pas en parfait accord avec les travaux antérieurs [6] qui ont montré que le lait de chamelle est plus pauvre en matière sèche et en matière protéique que celui de vache, cette différence peut être due à l'alimentation des animaux, aux conditions environnementales ainsi qu'au stade de lactation : fin de lactation [7].

Le lait de chamelle analysé est plus riche, essentiellement en matière protéique et matière sèche et légèrement en matière grasse, que celui de vache. Ces composantes montrent - comme décrit par Guliye et al [8]- des variations importantes selon le stade de lactation et l'espèce. Le lait camelin est plus riche en lactose (carbohydrate majeur du lait) que celui des bovins, ceci a été décrit par d'autres études [10]. La concentration du lactose varie durant la période de lactation : elle est faible à la naissance $(2,8 \%)$ mais augmente durant les premières 24 heures et atteint $5 \%$ tant que la quantité d'eau nécessaire est disponible [9].

La teneur en azote non protéique dans le lait de chamelle est significativement plus importante que celle du lait de vache, ces résultats sont en parfait accord avec ceux rapportés par la d'autres travaux $[10,11]$. Les fractions (NPN) présentent une importance biologique due à leur richesse en acides aminés acides ainsi qu'en vitamine B [12]. Ce qui augmente la valeur nutritive au lait de chamelle. 
Tableau 2 : Comparaison de la composition chimique du lait de vache et de chamelle

\begin{tabular}{|c|c|c|c|c|c|}
\hline \multirow[t]{2}{*}{ Paramètres } & \multicolumn{2}{|c|}{ Lait de chamelle (L.Ch) } & \multicolumn{2}{|c|}{ Lait de vache (L.V) } & \multirow{2}{*}{$\begin{array}{c}\text { Test de } t \text { : } \\
\text { Valeur de } \\
p\end{array}$} \\
\hline & Moyenne & Ecart-type & Moyenne & Ecart-type & \\
\hline Matière grasse $(g / L)$ & 37,5 & 8,95 & 32,5 & 9,118 & 0,217 \\
\hline Matière sèche $(\mathrm{g} / \mathrm{L})$ & 119,438 & 15,34 & 104,88 & 14,37 & 0,057 \\
\hline Cendres $(\mathrm{g} / \mathrm{L})$ & 7,5 & 7,75 & 6,67 & 1,76 & 0,397 \\
\hline Lactose $(\mathrm{g} / \mathrm{L}$ & 42.78 & 2,36 & 40,2 & 1,35 & 0,146 \\
\hline NPN $(\mathrm{g} / \mathrm{L})$ & 1,04 & 0,08 & 0,76 & 0,05 & $0,0016^{*}$ \\
\hline Protéines totales (g/L & 34,15 & 3,114 & 30,5 & 4,95 & 0,066 \\
\hline
\end{tabular}

$p^{*} \leq 0,05$ : La différence est significative entre les deux paramètres comparés; $p>0,05:$ La différence est non significative

\section{3-1-4. Composition en éléments minéraux}

La composition en éléments minéraux majeurs $(\mathrm{Na}, \mathrm{K}$, $\mathrm{Ca}$ et $\mathrm{Mg}$ ) déterminée par spectrométrie d'absorption atomique est représentée dans le Tableau 3.

Tableau 3: Composition en éléments minéraux des laits de vache et chamelle

\begin{tabular}{|l|l|l|l|}
\hline $\begin{array}{l}\text { Eléments } \\
\text { minéraux(g/L) }\end{array}$ & $\begin{array}{l}\text { Lait de Chamelle } \\
\text { (L.Ch) }\end{array}$ & $\begin{array}{l}\text { Lait de Vache } \\
\text { (L.V) }\end{array}$ & $\begin{array}{l}\text { Test T : valeur } \\
\text { de p }\end{array}$ \\
\hline $\mathrm{Na}$ & $0,47 \pm 0,055$ & $0,43 \pm 0,017$ & 0,094 \\
\hline $\mathrm{K}$ & $2,46 \pm 0,008$ & $1,50 \pm 0,18$ & 0,0017 \\
\hline $\mathrm{Ca}$ & $1,65 \pm 0,066$ & $1 \pm 0,060$ & 0,53 \\
\hline $\mathrm{Mg}$ & $0,07 \pm 0,029$ & $0,07 \pm 0,088$ & 0,065 \\
\hline $\mathrm{Zn}$ & $0,02 \pm 0,076$ & $0,02 \pm 0,056$ & 0,949 \\
\hline $\mathrm{Cu}$ & $0.3 \pm 0,05$ & $0.23 \pm 0,02$ & 0,597 \\
\hline
\end{tabular}

La composition minérale du lait de chamelle est plus riche en éléments minéraux majeurs $(\mathrm{Ca}, \mathrm{Mg}, \mathrm{Na}$, et $\mathrm{K})$ que celle du lait de vache comme était reporté par d'autres travaux $[13,14]$. Mais montrent toujours des différences entre les études effectuées $[10,15]$ concernant la concentration en ces minéraux ainsi que d'autres ( $\mathrm{Zn}, \mathrm{Cu})$. Cet 
intervalle de variation peut être du à la nature de l'élevage, l'alimentation, le stade de lactation ainsi qu'aux procédés d'analyses $[10,13]$

\section{3-2. Variations du pH et de l'acidité du lait à différentes températures}

Dans le but d'étudier le comportement du $\mathrm{pH}$ et de l'acidité (paramètres indispensables pour le suivi de la conservation du lait) des échantillons de lait déjà décrit, on a essayé de suivre l'évolution de ces deux paramètres à plusieurs températures : température ambiante, à 4 et à $-4^{\circ} \mathrm{C}$.

\section{3-2-1. A température ambiante $\left(25^{\circ} \mathrm{C}\right)$}

Les échantillons de lait frais sont mis à température du laboratoire $\left(25 \pm 2^{\circ} \mathrm{C}\right)$ après détermination du pH et de l'acidité à l'état frais. Ces deux paramètres sont mesurés le matin et l'après midi jusqu'à acidification du lait. Les résultats de cette étape sont illustrés dans la figure 1 .

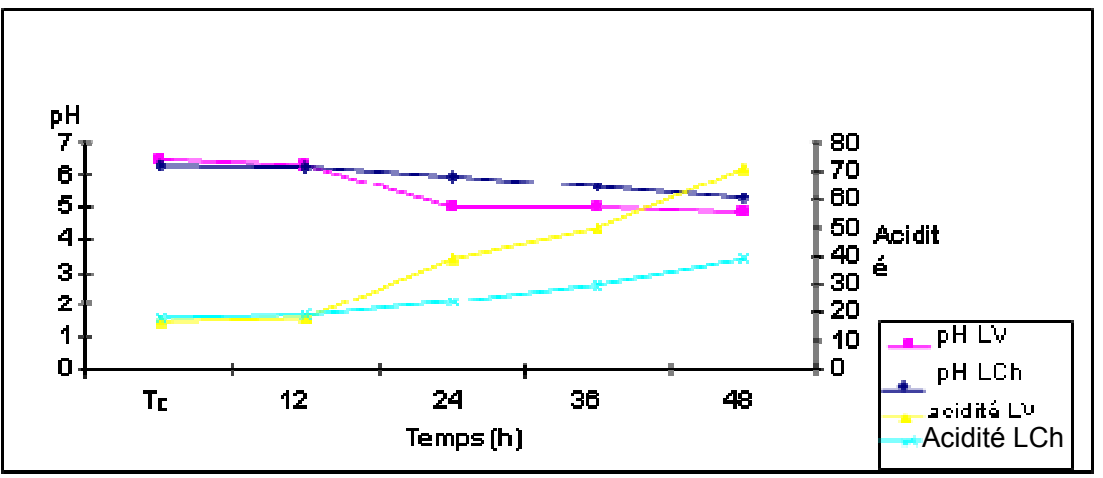

Figure 1 : Variation du pH et de l'acidité du lait de vache et de chamelle à température ambiante $\left(25 \pm 2^{\circ} \mathrm{C}\right)$

D'après la Figure $I$ si dessus on remarque que le lait de vache s'acidifie plus rapidement que le lait de chamelle et montre, à température ambiante, après 24 heures une augmentation importante de l'acidité $\left(39^{\circ} \mathrm{D}\right)$ accompagnée d'une diminution du $\mathrm{pH}(\mathrm{pH}=5)$.

Le lait de chamelle parait s'acidifiant lentement et atteint un $\mathrm{pH}=5,64$ et montre une acidité très inférieure $\left(39^{\circ} \mathrm{D}\right)$ à celle du lait de vache $\left(71^{\circ} \mathrm{D}\right)$ après $48 \mathrm{~h}$.

L'acidification lente du lait de chamelle peut être expliquée principalement par son pouvoir tampon [16]. 


\section{3-2-2. $A 4^{\circ} \mathrm{C}$}

Les échantillons du lait sont conservés dans le réfrigérateur à $4^{\circ} \mathrm{C}$ pour suivre l'évolution du pH et de l'acidité d'une façon journalière. Le suivi est arrêté lorsqu'on remarque une modification de l'aspect du lait : coagulation et odeur désagréable. Les résultats obtenus sont illustrés dans la figure 2.

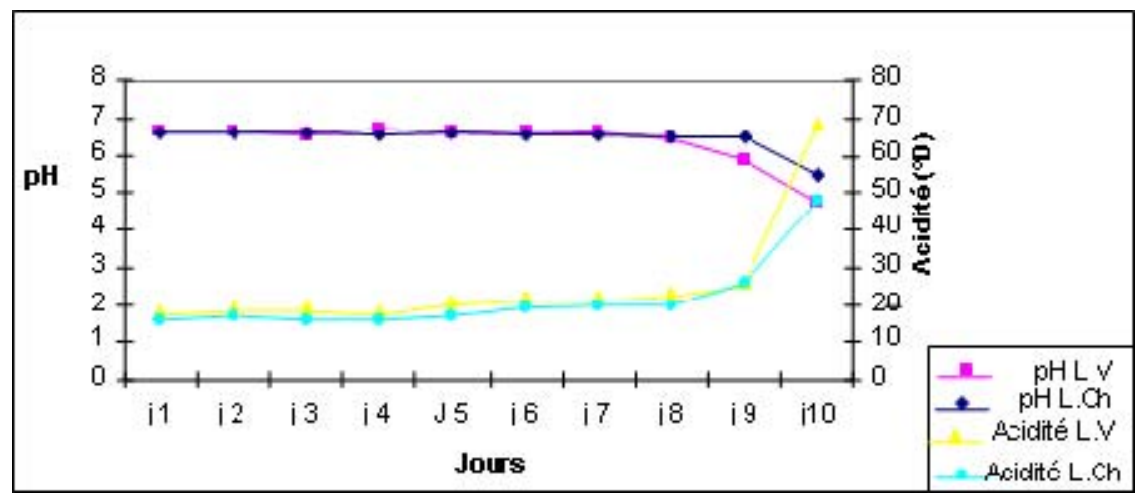

Figure 2 : Variations du pH et de l'acidité du lait de chamelle et vache à $4^{\circ} \mathrm{C}$

Après 7 jours de conservation à $4^{\circ} \mathrm{C}$, l'acidité moyenne des échantillons du lait de chamelle est dans les normes $\left(20^{\circ} \mathrm{D}\right)$ [17], correspondant à un pH moyen égal à 6,52. L'acidité du lait de vache commence à s'élever d'une façon remarquable dès le $5^{\text {ème }}$ jour $\left(22^{\circ} \mathrm{D}\right)[18]$ avec un pH égale à 6,6 .

Cette lente acidification du lait de chamelle peut être expliquée par l'émulsion de sa matière grasse (particularité des globules gras) ainsi qu'à la suspension colloïdale des protéines $[19,20,18]$

L'activité antimicrobienne du lait de chamelle est supérieure à celle du lait de vache (richesse en lysozyme et en peroxydase) ce qui peut maintenir sa stabilité et ralentir sa fermentation [21]. Ces résultats montrent que même à $4^{\circ} \mathrm{C}$ le lait de chamelle peut se conserver plus longtemps (au moins 7 jours) que le lait de vache qui perd son aspect naturel après 5 jours (Figure 2).

\section{3-2-3. $A-4^{\circ} \mathrm{C}$}

Congelé à $-4^{\circ} \mathrm{C}$ pendant trois semaines à partir du jour de prélèvement, les mêmes paramètres physiques des échantillons du lait sont analysés pour étudier leur stabilité à cette température de congélation. 
Les résultats sont représentés dans la figure 3 et montrent que les deux paramètres (pH et acidité) sont presque stables à la température de congélation pour les deux types de lait.

Ces deux paramètres présentent des valeurs normales pour le lait de vache ainsi que pour le lait de chamelle.

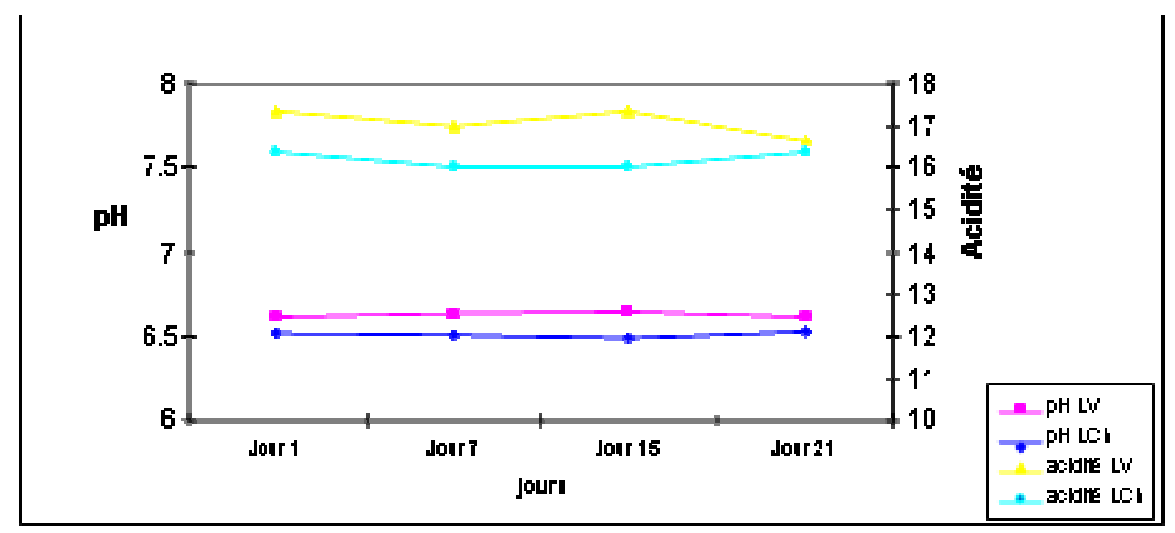

Figure 3 : Variations du pH et de l'acidité du lait de chamelle et de vache $\dot{a}-4^{\circ} \mathrm{C}$

La congélation peut donc être utilisée comme méthode efficace de conservation du lait (de vache ou de chamelle), permettant ainsi sa consommation plusieurs jours après la traite de l'animal.

La température de congélation utilisée est la plus efficace pour une meilleure conservation du lait. Cette résistance aux facteurs externes du lait de chamelle, se traduisant par une acidification lente à différentes températures, peut être expliquée par le pouvoir tampon, ainsi que par la composition particulière en protéines (teneur très faible en caséines Kappa) de ce lait $[5,3]$

La congélation du lait de chamelle constitue à cet égard un moyen de conservation du lait qui permet de répondre à l'un des soucis de l'Homme : rendre accessible la consommation du lait de dromadaire loin de son lieu de production.

\section{Conclusion}

Le lait de chamelle est un aliment spécifique par son aspect, sa composition et son comportement vis-à-vis aux changements des conditions du milieu.

En fait, le lait de chamelle à l'état frais est plus acide et moins dense que le lait bovin. 
Sa composition chimique est caractérisée par sa teneur importante en matière protéique ainsi qu'en vitamines $C$ et $B_{3}$, toutefois ces concentrations varient selon l'alimentation, le stade de lactation ainsi qu'aux conditions environnementales. Sa composition en éléments minéraux parait plus riche que le lait de vache surtout en Ca et Potassium.

La période de conservation du lait chamelle est fonction de la température du milieu, mais toujours plus longue que celle du lait de vache, elle est de plus que 24 heures à température ambiante, 7 jours à $4^{\circ} \mathrm{C}$ et plusieurs semaines sous congélation.

Comme l'utilisait les nomades ou les habitants des milieux arides, le lait de chamelle présente une grande valeur nutritive à l'état frais.

Néanmoins cette valeur nutritive et composition particulière peuvent se maintenir presque intactes par congélation, cette méthode peut satisfaire à l'un des soucis de l'Homme qui tente à trouver des procédés de conservation de ce lait pour différer dans le temps de sa consommation pour ne pas le limiter aux habitants des milieux arides.

\section{Références}

[1]- Z. Farah; Camel milk. Properties and products. SKAT. St.Gallen, Zurich, Switzerland.,(1996) 60-61

[2] - Afnor; Contrôle de la qualité des produits alimentaires. Lait et produits laitiers, (1993) Afnor(Ed), Paris, France.

[3] - Z. Farah; Composition and characteristics of camel milk. J. Dairy. Res., 60 (1993) 603-626.

[4] - G.M. Elbahay; Veterinary Medical Journal 8 (9) (1962) 7-18 in Farah. Z. Composition and characteristics of camel milk. J.Dairy.Res., 60 (1993) 603-626.

[5] - Z. Farah and M.R. Bachmann. Rennet coagulation properties of camel milk. Milch wissenshafte., 42 (1987) 689- 692.

[6] - M. Kammoun et Ellouze-Fourati. Evolution de la composition du lait de dromadaire en fonction de stade de lactation. In: CIHEAM- Options Méditerranéennes -6 (1989) $307-311$.

[7] - M.Kammoun et Ramet. Conservation et transformation du lait de dromadaire. In CIHEAM- Option Méditerranéennes -6 (1989) 229-231.

[8] - A. Guliye; R.Yagil and Hovell F.D; Milk composition of Bedouin camels under seminomadic production system. J. Camel. Pract. Res., 7 (2000) 209-212. 
[9] - R.Yagil and Z. Etzion. Effect of drought condition on the quality of camel milk. J. Dairy Res., 47 (1980) 159- 166.

[10] - I.H. Abu - Lehia. Physical and chemical characteristics of camel milk fat and its fractions. Food Chemistry., 34 (1989) 261- 272.

[11] - EK. Barbour, NH. Nabout, WM.Friedrichs and HM. ALNAKHIL. Inhibition of pathogenic bacteria by camel's milk: relation to whey lysozyme and stage of lactation. J.Food Protection., 47 (1984) 838- 840.

[12] - H. ZHANG, J.YAO, D ZHAO, H.LIU, M. GUO. Changes in chemical composition of Alxa Bactrian camel milk during lactation. J.Dairy. Sc., 88 (2005) 3402-3410.

[13] - M MEHAIA.Milk composition Wadha and Hamra camels in Saudi Arabia. Food Chemistry., 52 (1995) 115-122.

[14] - YASIN and WAHID. Pakistan camels, a preliminary survey. Agric., 8 (1957) 298.

[15] - A.M. GHORBAN and IZELDIN. Mineral content of camel milk and colostrum. Int. J. Food. Sci. Nutr., 52(1997) 283 - 287.

[16] - W.N. SAWAYA, J.K. KHALIL, A.AL-SHALHAT and H. AL-MOHAMED.Chemical composition and nutrition quality of camel milk., J. Food Sc., 39 (1984) 744-747.

[17] - H. El HATMI, T. KHORCHANI, M. HAMADI; M.ABDENEBI et H. ATtIA. Production et composition du lait de chamelle élevée dans le sud tunisien, Les filières laits en méditerranée: enjeux pour un futur durable. EAAP., 99 (2003) 62- 68.

[18] - N. Kherouatou. La micelle du lait camelin: Caractérisation physicochimique, rhéologique, biochimique et techno fonctionnelle. Thèse de Doctorat, Ecole National des Ingénieurs de Sfax, Tunisie., (2000) 201p.

[19] - H Attia, N. Kherouatou, N. Fakhfakh, T.Khorchani and N. Trigui. Dromedary milk fat: biochemical, microscopic and rheological characteristics. J. food Lipids., 07 (2000a) 95- 112.

[20] - H. Attia, N. Kherouatou, Nasri M and T. Khorchani. Characterization of the dromedary milk casein micelle and study of its changes during acidification, Lait.,80 (2000b) 503- 515.

[21]- El. El Agamy. Antibacterial and antiviral activity of camel milk protective proteins. J.Dairy. Res., 59 (1992) 169-175. 